\title{
CONSUMO DE ESTIMULANTES CEREBRAIS EM ACADÊMICOS DA ÁREA DA SAÚDE NA CIDADE DE PONTA GROSSA-PR
}

\section{CONSUMPTION OF BRAIN STIMULANTS IN HEALTH STUDENTS IN PONTA GROSSA-PR CITY}

\author{
Nícolas CORDEIRO'; Rodrigo Moreira Caetano PINTO²
}

1 - Acadêmico do Curso de Farmácia das Faculdades Ponta Grossa- Ponta Grossa-PR. 2 - Docente do Curso de Farmácia das Faculdades Ponta Grossa- Ponta Grossa-PR. Autor para correspondência: nicolascardie@gmail.com.

\section{RESUMO:}

Os estimulantes cerebrais são medicamentos capazes de aumentar a capacidade cognitiva através da estimulação cerebral. Há uma tendência de consumo dessas substâncias em indivíduos saudáveis com o intuito de intensificar o desempenho acadêmico, principalmente em jovens universitários. $O$ objetivo do presente estudo foi avaliar os hábitos de consumo de estimulantes cerebrais em acadêmicos da área da saúde das faculdades particulares na cidade de Ponta Grossa-PR. Para isso foram aplicados formulários compostos com 10 perguntas referentes ao tema, de forma anônima, no período de novembro de 2015. Participaram 793 acadêmicos, $70,9 \%$ do sexo feminino e $29,10 \%$ do masculino, com idade predominantemente entre 18 e 25 anos. Dentre os acadêmicos, $9,8 \%$ relatou consumir algum estimulante cerebral durante a vida (Ginkgo biloba, metilfenidato, modafinila ou piracetam). O medicamento mais consumido de forma off-label foi o Ginkgo biloba, $5,65 \%$, seguido do metilfenidato, $5,3 \%$. A forma mais citada do conhecimento sobre os estimulantes foi durante a graduação, $24,5 \%$. A modafinila é o medicamento mais desconhecido pelos estudantes. As motivações para o consumo foram o aumento da capacidade cognitiva, com o intuito de realizar tarefas específicas como trabalhos ou projetos. Dos usuários de Ginkgo biloba, cerca de 50,8\% não percebeu nenhum efeito significativo. Já quem fez uso de metilfenidato, $85 \%$ relatou uma melhora em sua capacidade de memorização e planejamento de ideias. O metilfenidato foi o medicamento que apresentou efeitos colaterais entre os usuários. O consumo de psicoestimulantes é considerado um comportamento de risco, principalmente pelo seu potencial elevado de efeitos adversos.

Palavras-chaves: Aumento cognitivo; nootrópicos; psicoestimulantes; off-label.

\section{ABSTRACT:}

The cerebral stimulant drugs are capable of enhancing the cognitive capacity through brain stimulation. There is a trend of consumption of these substances in healthy subjects in order to enhance academic performance, mainly university students. The aim of this study was to evaluate the consumption patterns of brain stimulants in the academic health of private colleges in the Ponta Grossa-PR city. For that were applied compounds forms with 10 questions on the topic, anonymously, from November 2015 participated 793 students, $70.9 \%$ were female and $29.10 \%$ male, aged mainly between 18 and 25 years. Among academics, $9.8 \%$ of students reported consuming some brain stimulant during life (Ginkgo biloba, methylphenidate, modafinil or piracetam). The most consumed off-label manner was 
the Ginkgo biloba drug, 5.65\%, followed by methylphenidate, $5.3 \%$. The most frequently cited form of the knowledge of stimulants was during graduation, $24.5 \%$. Modafinil is the most unknown medication by students. The motivations for consumption were increased cognitive ability, in order to perform specific tasks such as work or projects. Ginkgo biloba users, about $50.8 \%$ did not notice any significant effect. Who have made use of methylphenidate, $85 \%$ reported an improvement in their ability to memorize and planning ideas. Methylphenidate was the medicine that had side effects among users. The consumption of psychostimulants is considered a risk behavior, especially for its high potential for adverse effects.

Keywords: Cognitive enhancement; nootropics; psychostimulants; off-label.

\section{INTRODUÇÃO}

A busca do ser humano por uma vantagem em relação aos outros é antiga, e

remota ao uso de anfetaminas por soldados na $2^{\underline{a}}$ Guerra Mundial, onde esses medicamentos eram usados com o intuito de mantê-los alertas e disponíveis para a batalha. (MOHAMED; SAHAKIAN, 2012; SWANSON; WIGAL; VOLKOW, 2011).

A idéia de aumentar a memória, concentração e o aprendizado de forma rápida e definitiva nos acompanha como humanidade há milênios, e está intimamente relacionado com a evolução. O termo "aperfeiçoamento cognitivo" surgiu no inicio dessa década para relatar a possibilidade de um fármaco produzir, de forma artificial, uma maior capacidade de retenção de memória, concentração e planejamento de tarefas, características já presentes em pessoas isentas de transtornos de déficit intelectual. (HYMAN, 2011; SAHAKIAN et al., 2015).

O aperfeiçoamento ou aprimoramento cognitivo pode ser definido como a ampliação das capacidades básicas cerebrais, através da melhoria ou aumento dos sistemas de processamento de informações internas e externas. Desta forma, o melhoramento leva o indivíduo a um estado maior de concentração e raciocínio, conceitos inerentes às atividades intelectuais. (DESANTIS; HANE, 2010).

Os estimulantes cerebrais, nootrópicos ou psicoestimulantes são substâncias químicas capazes de alterar ou aumentar o desempenho intelectual cerebral através da estimulação ou inibição de certos neurotransmissores. Esses agentes podem ocorrer naturalmente ou serem derivados sinteticamente, podendo atuar de forma excitatória ou suprimindo ações inibitórias. (SANIOTIS et al., 2014; SILVA, 2010; TEIXEIRA, 2007).

O aprimoramento intelectual recebeu considerável atenção científica ao longo da ultima década. Somente agora a complexidade cerebral está sendo elucidada, sobretudo 
os conceitos básicos sobre a cognição. Numerosos debates em relação à ética da melhoria cognitiva estão ganhando foco, principalmente no uso não prescrito, ou chamado uso offlabel de estimulantes cerebrais por estudantes universitários. (HILDT; LIEB; FRANKE, 2014; VRECKO, 2013,2015).

Quase sem exceção, tais discussões sugerem que estes medicamentos permitem melhorar o desempenho acadêmico através dos efeitos positivos sobre os processos cerebrais. Porém, não há na atualidade um medicamento proposto somente para esses fins se não nas doenças neurodegenerativas como o Alzheimer ou no transtorno de déficit de atenção e hiperatividade. (TDAH) (HUSAIN; MEHTA, 2011; SMITH; FARAH, 2011).

A literatura contemporânea sobre o aprimoramento acadêmico tem-se concentrado principalmente em quatro substâncias farmacologicamente ativas: o metilfenidato (RITALINA® e CONCERTA®), modafinila (PROVIGIL® e STAVIGILE®), ambos com controle especial no Brasil (Portaria 344/1998 ANVISA-MS), e nos fármacos piracetam, comercializado no Brasil como a especialidade farmacêutica NOOTROPIL $\circledast$ e preparações contendo extratos padronizados de Ginkgo biloba. (FILHO; FAKOURY; FERRY, 2010; SINGH; BARD; JACKSON, 2014).

Embora o Ginkgo biloba e o piracetam apresentem mecanismo de ação distintos da modafinila e do metilfenidato, por conveniência de estudo e de pesquisa, a literatura adequou esses dois medicamentos a categoria de estimulantes cerebrais, pois invariavelmente seu consumo está relacionado à melhoria dos processos de aprendizado, memória e atenção. (URBAN; GAO, 2014).

O metilfenidato é atualmente o medicamento mais prescrito para o TDAH. Este fármaco atua no sistema nervoso central (SNC) impedindo a recaptação de dopamina e noradrenalina, fazendo com que esses neurotransmissores permaneçam mais tempo disponíveis nas fendas sinápticas. Promove efeito estimulante, aumentando a vigília e a concentração, provavelmente em regiões cerebrais críticas relacionadas ao distúrbio. (CARVALHO; BRANT; MELO, 2014; FREESE et al., 2012; ORTEGA et al., 2010; PEREIRA; BEL, 2010; REPANTIS et al., 2010).

A RITALINA® é o estimulante mais consumido no mundo e também o mais desviado para uso recreativo. O Brasil é o segundo maior consumidor, ficando atrás somente dos EUA. No ano de 2012, o Sistema Nacional de Gerenciamento de Produtos Controlados (SNGPC) relatou uma alta no consumo desse medicamento, aproximadamente $28,2 \%$ de caixas vendidas a mais na comparação com o ano de 2009. Os dados levantados não revelam a proporção do consumo prescrito e off- label. (BRASIL, 
2012; CUTLER, 2014; DECOTELLI; BOHRER; BICALHO, 2013).

O metilfenidato é considerado um medicamento seguro em doses terapêuticas. Os principais efeitos colaterais que tendem a ocorrer em até $50 \%$ dos usuários, com recomendação médica de uso, são hipertensão arterial sistêmica, redução do apetite e insônia. Há também uma associação do uso desse estimulante e a exacerbação de doenças mentais subjacentes, principalmente em pacientes sem diagnóstico de déficit de atenção ou hiperatividade. (ALOE et al., 2010; COUTO; JUNIOR; GOMES, 2010; SANIOTIS et al., 2014).

O extrato de Ginkgo biloba contém inúmeros glicosídeos, e embora o mecanismo farmacológico exato não seja totalmente compreendido, a ação combinada desses compostos parece promover um aumento do suprimento sanguíneo cerebral, além de reduzir a ação de radicais livres. (FILHO; FAKOURY; FERRY, 2010; SANDERSLEBEN et al., 2014).

Segundo Laws, Sweetnam e Kondel (2012), em um estudo com cerca de 1000 voluntários saudáveis, o Ginkgo biloba não resultou em aumento cognitivo significativo. Os efeitos mais pronunciáveis continuam sendo em pacientes com doenças neurodegenerativas e distúrbios circulatórios. Seu uso pode estar associado à potencialização de fármacos anticoagulantes, vertigens e cefaleias. (BALBINO; DIAS, 2010; IHL, 2012, 2013).

A modafinila é uma droga estimulante classificada como atípica devido ao seu mecanismo de ação e estrutura química serem distintos dos demais estimulantes. Sua ação está relacionada com o bloqueio da recaptação de serotonina e dopamina, e consequentemente, aumento da disponibilidade desses neurotransmissores responsáveis pelo estado de vigília e concentração. (ALOE et al., 2010; GIORELLI et al., 2012).

É aprovado no Brasil para o tratamento de sonolência excessiva associada à narcolepsia e na apneia do sono. Diversos estudos afirmam que a modafinila pode melhorar aspectos cognitivos, nas atividades que envolvam atenção e planejamento de ações. Em pessoas saudáveis, pode ser uma alternativa relevante aos efeitos deletérios dos trabalhos em turnos e prolongados. (GRADY et al.,2010; MULLER et al., 2013).

Apesar de prometer aumentar potencialmente a concentração, no curto prazo a modafinila é conhecida por produzir náuseas, êmese, diarreia, dispepsia, dor de cabeça, insônia e ansiedade. Suas complicações a longo prazo em adultos saudáveis, em grande parte, são desconhecidas. (BATTLEDAY; BREM, 2015; KELLEY et al., 2012).

O piracetam, o protótipo das chamadas drogas "nootrópicas" é usado em muitos 
países para o tratamento de disfunção cognitiva no envelhecimento e lesões cerebrais. Recentes análises de estudos clínicos forneceram evidências convincentes para a eficácia do piracetam em um grupo diversificado de indivíduos idosos com comprometimento intelectual. (KURZ et al., 2010; LEUNER et al., 2010).

Os efeitos benéficos do piracetam são normalmente associados em condições como o envelhecimento, hipóxia, privação de glicose, ferimentos ou danos dos radicais livres, pois aumenta a fluidez cerebral, o suprimento de glicose e oxigênio. Apesar do uso frequente entre jovens, não há indícios que justifiquem o uso em pessoas saudáveis. (ALKURAISHY et al., 2014; MALYKH; SADAIE, 2010).

Nos estudos realizados na Europa, observou-se um aumento em relação ao uso de estimulantes de forma off-label. Na França, o segundo estudo na área, entre 206 estudantes, 139 (67,4\%) declararam ter consumido pelo menos um nootrópico nos últimos 12 meses. No Reino Unido e na Irlanda, 877 acadêmicos participaram de uma pesquisa online e, cerca de $9,4 \%$ já utilizaram alguma substância para aumento cognitivo. (FRANCHI; MACGREGOR; FOND, 2014; SINGH; BARD; JACKSON, 2014).

$\mathrm{Na}$ Suíça, 13,8\% dos estudantes universitários indicaram que haviam usado psicoestimulantes sem prescrição médica pelo menos em uma ocasião. Nos Estados Unidos, através de uma revisão de literatura, as pesquisas indicam que o uso sem prescrição de estimulantes é de $35 \%$ em indivíduos em idade universitária. Os motivos relatados para o uso de estimulantes sem prescrição incluem aumentar a concentração e melhorar o estado de alerta. (MAIER et al., 2013; WILENS et al., 2008).

Respectivamente, aceita-se que informalmente essa prática no Brasil acontece, mas os estudos referentes ao tema apontam uma realidade evidenciada de outros países. (BARROS; ORTEGA, 2011).

Um dos poucos artigos e pioneiro no país, debate sobre o uso de metilfenidato em estudantes de medicina da Universidade Federal da Bahia. Dos pesquisados, 186 estudantes, 8,6\% relataram o uso em algum momento da vida universitária com intuito de melhorar o rendimento acadêmico. (CRUZ et al., 2011).

A automedicação é uma prática comum e crescente na atual sociedade. A inerente vontade de satisfazer uma necessidade pessoal ao passo de apenas utilizar uma pílula, leva muitos jovens a práticas inseguras. Quando se tratando de medicamentos não prescritos, ou off-label o problema torna-se mais grave, pois evidentemente os medicamentos consumidos não foram propostos para esses fins. (AQUINO; BARROS; SILVA, 2010; ARRIA; DUPONT, 2010). 
O uso indiscriminado de psicoestimulantes por universitários que não apresentam indicação é uma questão em foco internacional. Os poucos estudos realizados no Brasil referente à prática induzem a uma busca mais aprofundada dos hábitos existentes. $\mathrm{A}$ análise e compreensão são questões imprescindíveis para ações em saúde que envolva tais medicamentos. (PASQUINI, 2013; PESSANHA; MOTA, 2014).

O presente trabalho teve como objetivo avaliar os hábitos de consumo de estimulantes cerebrais entre acadêmicos da área da saúde no município de Ponta Grossa.

\section{MATERIAL E MÉTODOS}

Os locais de estudo foram as instituições de ensino superior privadas de Ponta Grossa que oferecem cursos na área da saúde, sendo eles: Educação Física, Enfermagem, Farmácia, Fisioterapia, Fonoaudiologia, Nutrição, Odontologia e Radiologia, com aproximadamente 1100 alunos matriculados, segundo dados das secretarias respectivas.

Foi utilizado como instrumento para coleta de dados um formulário composto por 10 perguntas (apêndice A), baseado e adaptado de The Smart Drugs Study (SINGH; BARD, 2012).

O universo amostral foram todos os acadêmicos devidamente matriculados nas instituições de ensino superior (IES) pesquisadas, no período de novembro de 2015 $(n=793)$, presentes nas datas de coleta dos formulários, de ambos os gêneros e maiores de 18 anos. Trata-se de uma pesquisa transversal descritiva, sendo estudo de campo de abordagem qualitativa.

Foram analisadas as seguintes variáveis: conhecimento em relação aos medicamentos citados no formulário de pesquisa, relação do consumo e resultados observados quando aplicado. Foram respeitados todos os aspectos éticos, seguindo a resolução 466/12 do Conselho Nacional de Ética em Pesquisa com Seres Humanos, sendo o projeto apreciado pelo Comitê de Ética em Pesquisa das Faculdades Ponta Grossa.

Após o aceite através da assinatura do termo de consentimento livre e esclarecido, (TCLE), os formulários forem entregues e respondidos, sem intervenção dos investigadores e de forma anônima.

As respostas que compuseram os formulários foram analisadas antes da tabulação, a fim de apontar possíveis faltas de preenchimento. Logo, os dados foram enviados para planilhas online do Google Docs® e exportadas na sequência para o programa Microsoft Excel $2010 \AA$, que permitiu a análise dos resultados que compõem as tabelas e gráficos. 
O critério de exclusão foi a comunicação de ser portador (a) de algum distúrbio que justificasse o uso prescrito, como a narcolepsia ou TDAH.

\section{RESULTADOS E DISCUSSÃO}

Dos 793 participantes, $71 \%$ eram do sexo feminino, enquanto $29 \%$ do masculino. A idade predominante foi de 18 a 25 anos, $66 \%$ do total, distribuídos entre os 5 anos da duração regular da graduação, abrangendo o total da população universitária.

Nenhum dos participantes relatou possuir diagnóstico para uso das substâncias referidas. Logo, as características de consumo encaixam-se no delineamento da pesquisa, que visa o enfoque no uso sem prescrição. A distribuição por curso e participação é demonstrada na Tabela 1.

Tabela 1- Estratificação da população amostral por curso

\begin{tabular}{lcc}
\hline \multicolumn{1}{c}{ Curso } & Participação & Porcentagem \\
\hline Educação Física & 194 & 24,5 \\
Enfermagem & 71 & 9,0 \\
Farmácia & 114 & 14,4 \\
Fisioterapia & 57 & 7,2 \\
Fonoaudiologia & 61 & 7,7 \\
Nutrição & 92 & 11,6 \\
Odontologia & 118 & 14,9 \\
Radiologia & 86 & 10,8 \\
Total Geral & 793 & $100 \%$ \\
\hline
\end{tabular}

Fonte: O Autor (2015).

Dentre o universo amostral, 9,8\% dos estudantes $(n=78)$ já utilizou algum estimulante cerebral de forma off-label. Esses acadêmicos são em sua maioria do sexo feminino, $74,3 \%(n=58)$, com idade entre 18 a 25 anos, 57,6\% $(n=45)$ cursando entre $01^{\circ}$ e $2^{\circ}$ ano, $55,1 \%(n=43)$. A apresentação desses dados confirma a hipótese de que o 
consumo de estimulantes cerebrais sem prescrição médica está presente entre universitários brasileiros.

A predominância do consumo entre os estudantes do sexo feminino com idade entre 18 a 25 anos é justificável, visto que é a maioria entre os cursos de graduação respectivos. Essa tendência também é mencionada nos estudos de Aquino, Barros e Silva (2010) e Graça (2013).

De um modo geral, os resultados apreciados encontram-se de acordo com as pesquisas internacionais realizadas com os mesmos medicamentos. No estudo de Singh, Bard e Jackson (2014) a incidência de consumo ficou em 9,4\% no Reino Unido e Irlanda. Teter et al. (2010) apresentou uma prevalência de 5,9\% de uso sem prescrição nos estudantes americanos. Em contra partida, Franchi, MacGregor e Fond (2014) verificou que $67,4 \%$ dos estudantes franceses fizeram uso, enquanto Franke et al.(2011) observou apenas $0,8 \%$ na Alemanha.

O primeiro contato com o medicamento foi mencionado de acordo com a Figura 1. As principais formas de informação sobre os estimulantes foram no decorrer da graduação $24 \%(n=194)$ e a internet $18 \%(n=143)$.

De acordo com Aquino, Barros e Silva (2010), há uma relação construída entre o conhecimento adquirido e a tendência da automedicação. Sobre isso, decai o fato de que a proximidade com os medicamentos impõe uma maior utilização sem prescrição. Fato que reforça a importância de implementar medidas preventivas nas faculdades, evitando assim comportamentos de risco.

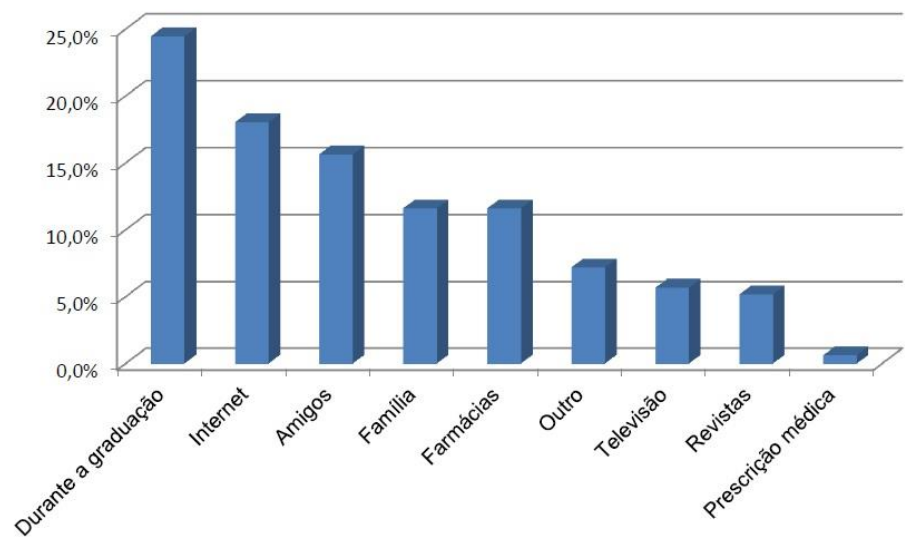

Fonte: O Autor (2015)

Figura 1 - Principais fontes de informação sobre os estimulantes cerebrais 
Outra fonte importante de informação citada pelos estudantes foi a internet, que é também verificado por Barros e Ortega (2011). Fóruns de discussão, redes sociais e sites de notícias contribuem muitas vezes incentivando o consumo, suprindo dúvidas pertinentes de forma leiga e equivocada.

O que de fato é comprovado, pois a maioria dos usuários indica o consumo de estimulantes em um período anterior a graduação, o que é debatido a seguir. Sendo assim, é importante que os estudantes obtenham a informação adequada, através de um profissional da área.

As motivações para o consumo destas substâncias estão elencadas na Figura 2. De acordo com os dados obtidos, $82 \%(n=64)$ dos estudantes fizeram uso de psicoestimulantes com intuito de aumentar a capacidade cognitiva.

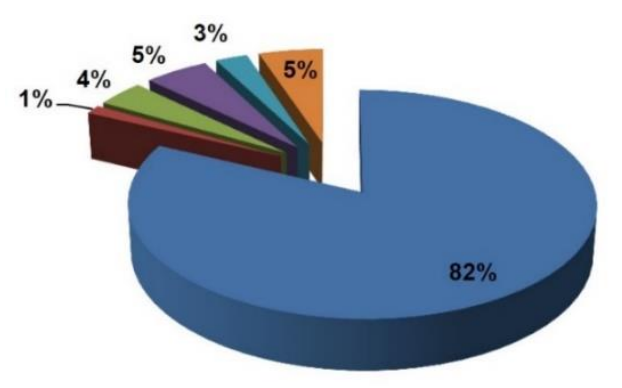

Fonte: O Autor (2015)

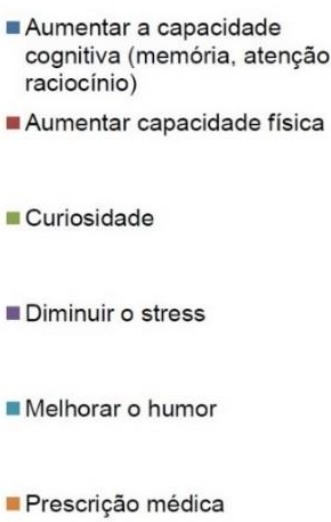

Figura 2 - Principais motivações para o consumo de estimulantes

Sendo o aumento da capacidade cognitiva a principal motivação para o consumo, a hipótese inicial é confirmada e confere com a tendência observada por outros autores. Graça (2013), Teter et al.(2010), Maier et al.(2013) e Partridge et al. (2013) reportam motivos idênticos. Advokat, Guidry e Martino (2008) incluem melhorar o desempenho intelectual e aumentar as notas como alguns fatores.

Graça (2013) observa esse fato como uma tendência de comportamento atual. As dificuldades que o ensino superior exige podem levar os jovens ao uso de substâncias que permitem conciliar as atividades acadêmicas com a vida pessoal.

De acordo com a Figura 3, dos estudantes que indicaram como motivação para o consumo aumentar a capacidade cognitiva, $47 \%(n=31)$ afirmou fazer uso com o intuito de 
auxiliar no estudo, ao aumentar a memória e a concentração, seguido da conclusão de trabalhos específicos como projetos ou pesquisas, cerca de $17 \%(n=11)$.

Teter et al.(2010) já havia relacionado em seus estudos esta prevalência. Citou ainda haver um consumo predominantemente esporádico, isto é, antes de avaliações importantes, concursos e outros. Não descartando a hipótese do uso contínuo e prolongado, o que pode ser uma tendência nos estudantes pesquisados.
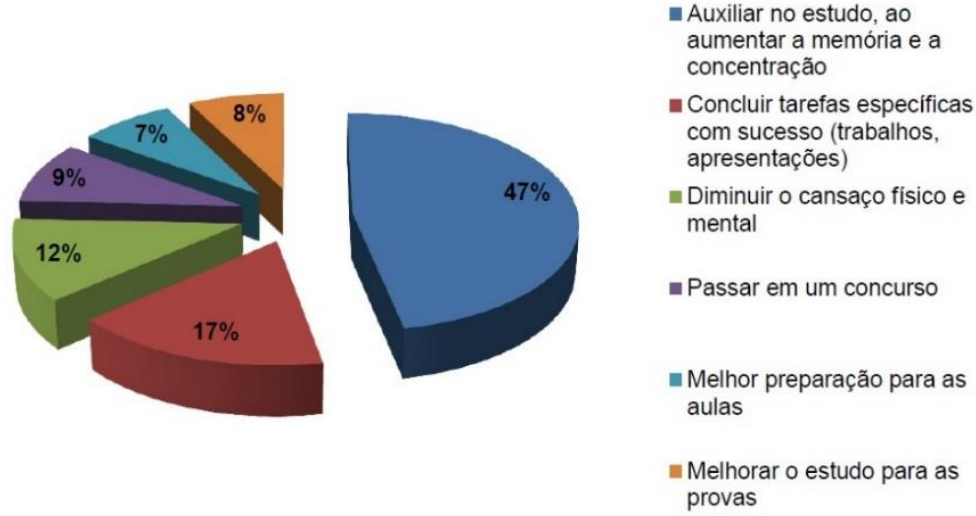

Fonte: O Autor (2015)

Figura 3 - Motivações específicas para o aumento da capacidade cognitiva

A maioria dos acadêmicos indicou que iniciou o consumo de Ginkgo biloba, piracetam, metilfenidato e modafinila antes de ingressar na faculdade, $71 \%(n=56)$, conforme a Figura 4.

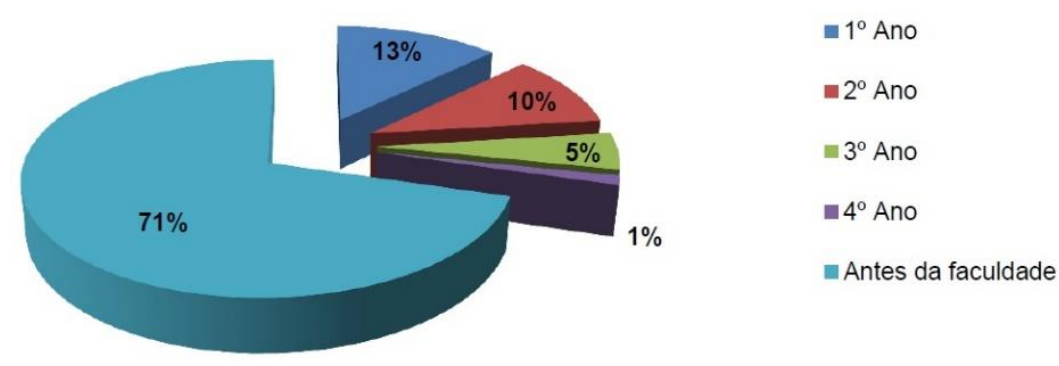

Fonte: O Autor (2015)

Figura 4 - Início do consumo de estimulantes

Segundo Filho,Fakoury e Ferry (2010) o Ginkgo biloba, piracetam, metilfenidato e a modafinila estão entre os medicamentos mais usados para fins não terapêuticos no mundo. Nesse sentido, a média de consumo, conhecimento e efeitos é elencada com a 
tendência geral e específica, para cada medicamento citado.

A média de consumo e conhecimento do Ginkgo biloba é exemplificada na Figura 5. De acordo com os dados levantados, 5,65\% $(n=44)$ já consumiram este medicamento em algum momento da vida.

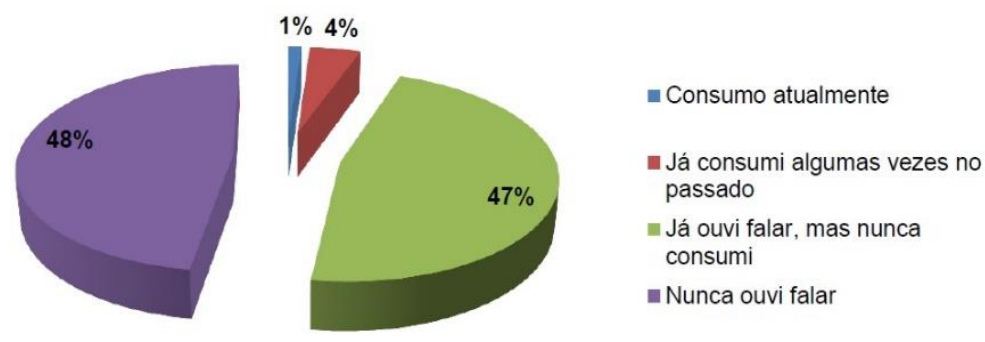

Fonte: O Autor (2015)

Figura 5 - Relação de conhecimento e consumo de Ginkgo biloba

O Ginkgo biloba teve a maior prevalência de consumo entre todos os medicamentos pesquisados (Ginkgo biloba, piracetam, metilfenidato e modafinila). Nos dias atuais, é um dos fitoterápicos mais consumidos no Brasil. De fácil acesso, possui uma gama de produtos com seu princípio ativo, como chás e cápsulas. (LEITE; BRANCO, 2010).

Pasquini (2013) observou prevalência de 3,4\% de consumo de Ginkgo biloba entre universitários brasileiros. Verificou também uma associação com metilfenidato e piracetam no intuito de potencialização dos efeitos estimulantes.

Apesar da facilidade de compra e da promessa de melhoria das faculdades intelectuais, a maioria dos acadêmicos usuários admitiu não notar qualquer efeito pronunciável, aproximadamente $51 \%(n=21)$, conforme Figura 6 .

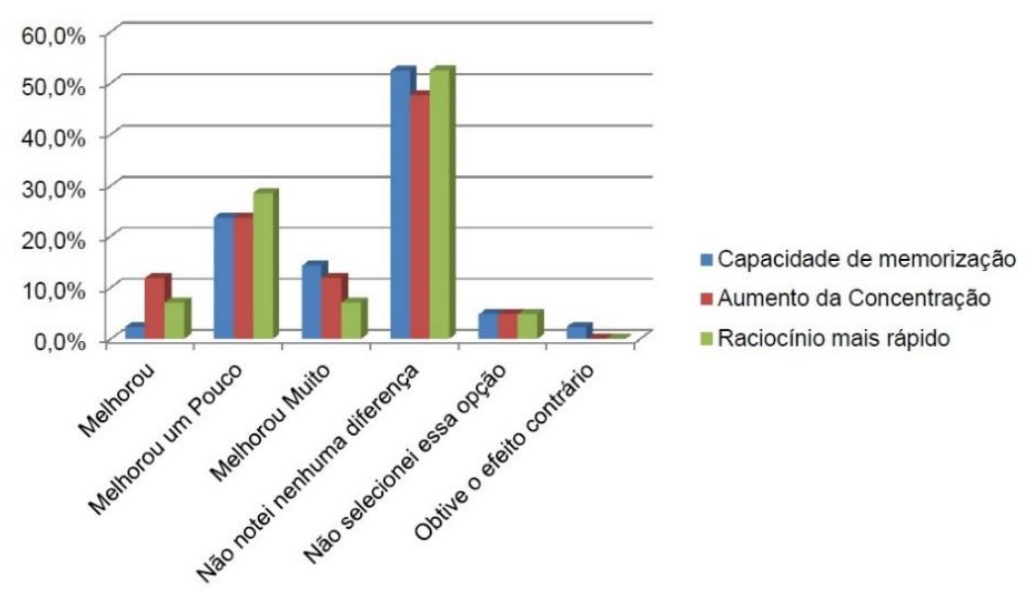

Fonte: O Autor (2015)

Figura 6 - Efeitos atribuídos ao uso off-label de Ginkgo biloba 
O extrato de Ginkgo biloba é prescrito geralmente em síndromes neurodegenerativas, como modulador e atenuante aos efeitos deletérios da velhice. É indicado nos problemas de concentração, zumbidos, cefaléias e particularmente, nas alterações do desempenho cerebral. Porém, sua ação mais pronunciável continua sendo em relação à agregação plaquetária, pois é um antagonista do receptor do fator ativador de plaquetas. (PAF) (FILHO et al., 2007).

Os resultados apurados no uso do Ginkgo biloba estão em acordo com Laws, Sweetnam e Kondel (2012). Segundo o respectivo estudo, após etapas de testes envolvendo raciocínio lógico, não houve uma contribuição significativa que justificasse o uso em indivíduos saudáveis. Apesar de a literatura ser unânime afirmando desconhecer os efeitos cognitivos, diversos laboratórios afirmam o contrário, sem estudos clínicos que comprovem essa suposição.

Os nootrópicos mais desconhecidos aos estudantes são a modafinila 82\% ( $n=645)$ e o piracetam $74 \%(n=585)$. Apenas um estudante relatou o consumo de modafinila, enquanto $2 \%$ $(n=10)$ admitiu o uso de piracetam.

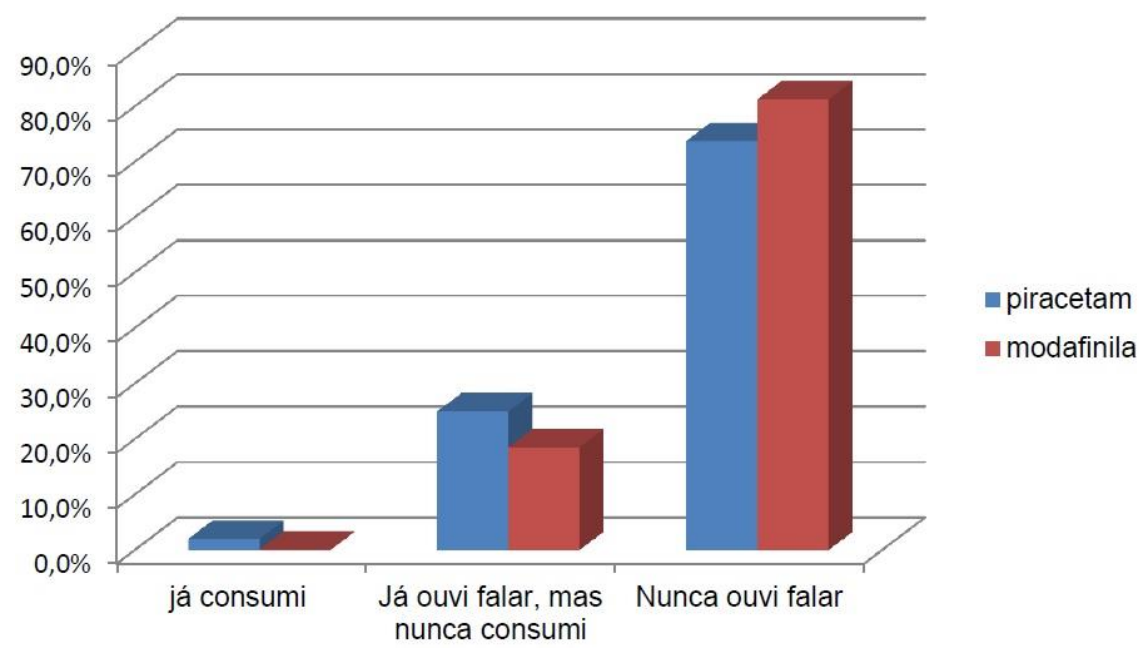

Fonte: O Autor (2015)

Figura 7 - Relação do conhecimento e consumo da modafinila e piracetam

Graça (2013) apresenta uma tendência semelhante nos estudantes de Portugal. Em seu estudo, 65\% dos participantes desconheciam o piracetam e $67 \%$ a modafinila. $O$ consumo do piracetam relatado em seu estudo teve uma prevalência de $11 \%$. Já Franchi, MacGregor e Fond (2014) relataram prevalência de 5,8\% de consumo de piracetam nos estudantes franceses.

A modafinila é um medicamento relativamente desconhecido, não só no Brasil, mas como em boa parte do mundo. Trata-se de um medicamento novo, com poucas citações 
na literatura, mas com um grande potencial de pesquisa. Segundo Maier et al. (2013) a prevalência de uso é $0,3 \%$ entre os universitários suíços, enquanto Franchi, MacGregor e Fond (2014) não referiu consumo entre os universitários franceses.

O único estudante que fez uso de modafinila admitiu melhorar o humor como motivação, citando aumento discreto no raciocínio, concentração e memorização. Kelley et al.(2012) em seus estudos relatou melhora discreta no uso off-label do modafinil em indivíduos saudáveis. Constatou também haver uma grande lacuna na literatura avaliando os efeitos do uso contínuo e prolongado.

De acordo com a Figura 8, dentre os acadêmicos que fizeram uso de piracetam, $50 \%(n=5)$ não relatou diferença cognitiva.

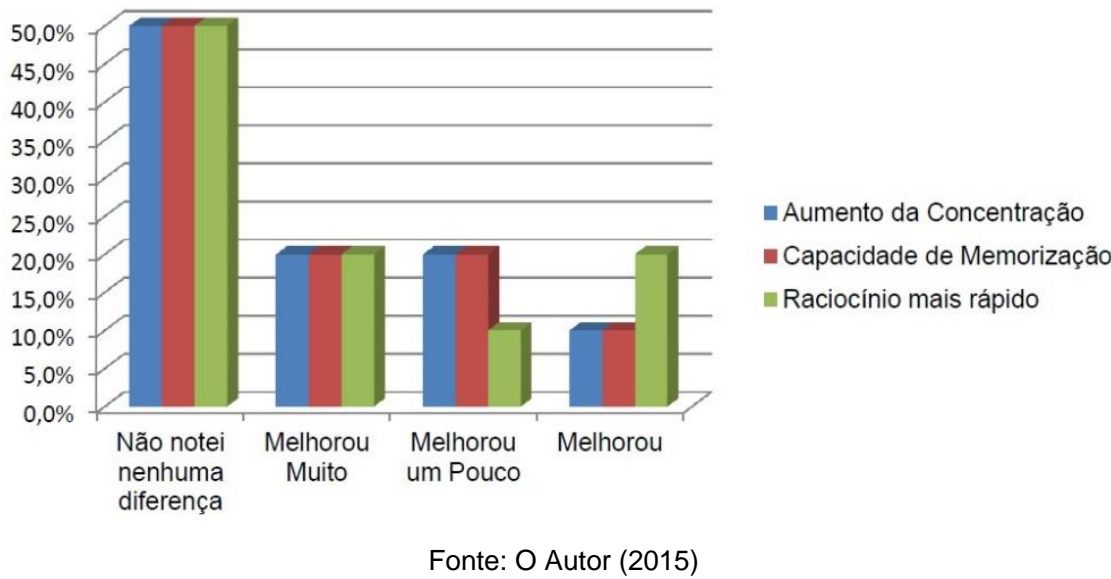

Figura 8 - Efeitos atribuídos ao uso off-label do piracetam

Os dados obtidos concordam com Leuner et al. (2010) que não relatou melhora cognitiva significativa. Diferentemente de Alkuraishy, Mahdi e Ashoor (2012), que apresentou um estudo relatando efeitos estimuladores do piracetam no cérebro. De acordo com esse mesmo autor, os resultados positivos foram observados com o uso contínuo e prolongado, cerca de 6 a 12 semanas.

O que pode ser percebido é o consumo não contínuo relatado pelos estudantes, sendo que $70 \%$ relatou já ter consumido no passado de forma ocasional, e os outros $30 \%$ consumem atualmente também de forma ocasional. Pode haver uma relação causal entre os efeitos atribuídos ao uso, com o tempo relativamente curto de consumo.

Conforme mostra a Figura $9,5,3 \%$ dos estudantes $(n=42)$ já consumiram metilfenidato em algum momento da vida e $81,97 \%$ ( $n=650)$ têm conhecimento do medicamento apresentado. 

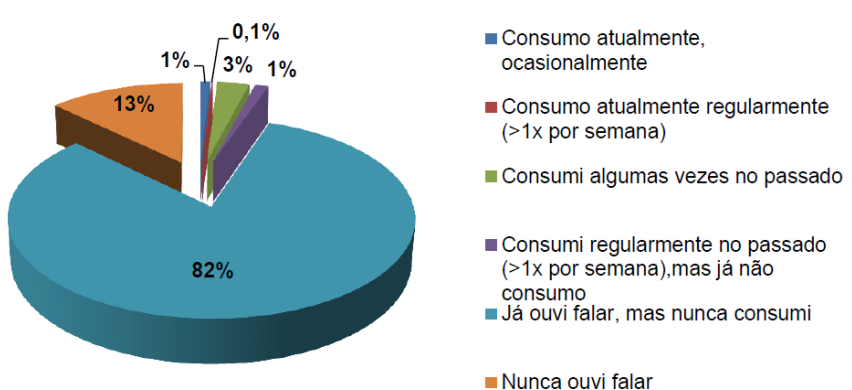

Fonte: O Autor (2015)

Figura 9 - Relação do consumo e conhecimento do metilfenidato

A RITALINA® é o psicoestimulante mais consumido de forma off-label no mundo. A literatura relacionada e este medicamento é vasta e bem detalhada, com isso observase um maior conhecimento por parte dos estudantes (82\%). A média de consumo de $5,3 \%$ apresenta um número expressivo, levando em conta a dificuldade de aquisição, já que apresenta uma substância com controle especial.

Cruz et al. (2011) reporta uma prevalência de consumo do metilfenidato de $8,6 \%$ entre estudantes de medicina de Porto Alegre, enquanto Pasquini (2013) relatou uso em cerca de $44,1 \%$ dos estudantes paulistas. Pessanha e Mota (2014) afirmou que $60 \%$ dos acadêmicos pesquisados já utilizou o metilfenidato durante a faculdade.

Em estudantes universitários americanos, Cutler (2014) relatou $9 \%$ de uso off- label enquanto Desantis e Hane (2010) encontrou prevalência de 34\% no uso de metilfenidato. Não há uma tendência universal de consumo, pois os contextos universitários diferem de uma região para outra.

Conforme a Figura 10, o metilfenidato foi o medicamento que mais apresentou melhoria da capacidade cognitiva (memória, raciocínio e concentração), em comparação com a Ginkgo biloba, modafinila e piracetam. Dos estudantes que relataram o consumo $(n=42), 85 \%$ referiu melhora nas propriedades cognitivas.

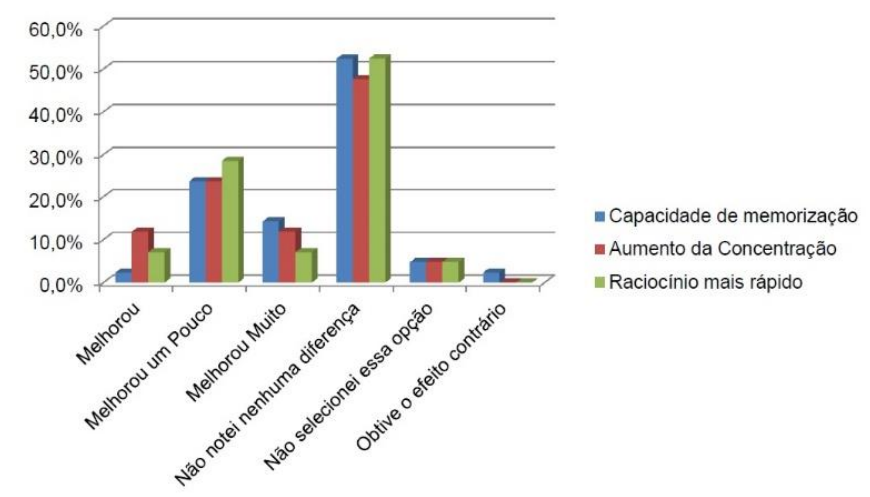

Fonte: O Autor (2015)

Figura 10 - Efeitos atribuídos ao uso off-label de metilfenidato 
Neste contexto, Vrecko (2013), Hildt, Lieb, e Franke (2014) compactuam da mesma análise em relação a RITALINA®, baseado em pesquisas com pessoas saudáveis. Segundo esses autores, a cognição pode ser alterada com o uso de metilfenidato. Smith e Farah (2011) ainda sugere melhoria na consolidação da memória, o que é um fator determinante para assimilar novos conhecimentos.

Diferentemente de outros nootrópicos como o piracetam, o metilfenidato é um psicoestimulante do grupo das anfetaminas e é classificado como uma droga psicotrópica, que pode causar dependência. É rapidamente absorvido após a administração oral, atingindo a concentração plasmática máxima em cerca de $2 \mathrm{~h}$. Seus efeitos são percebidos de imediato, e podem permanecer por horas. (PESSANHA; MOTA, 2014).

Conclui-se então que, mesmo com o uso ocasional relatado por alguns acadêmicos, o metilfenidato permite aprimorar o estado de concentração, memória e aprendizado, o que é observado também por Franchi, MacGregor e Fond (2014).

Do universo total de usuários $(n=78), 5 \%$ dos acadêmicos $(n=4)$ observaram aumento do estresse e declínio da sensação de bem estar como efeito atribuído ao uso de metilfenidato. Fato que baseia-se pela elevação do nível de atividade dopaminérgica, causando taquicardia e ansiedade. (CARNEIRO et al., 2013).

A literatura acadêmica apresenta diversos pontos de vista em relação ao uso de estimulantes cerebrais em pessoas saudáveis. A ética do melhoramento cognitivo é relatada por Forlini e Racini (2009), que ressaltam pontos de vista distintos em diversos autores. Há quem defenda que os psicoestimulantes, em sua maioria, são drogas seguras, e a utilização em indivíduos saudáveis é uma escolha pessoal.

Em oposição, vários autores defendem que seria uma opção antiética e uma atitude seletiva aumentar a concentração e memória, sem ao menos saber os efeitos que essas drogas podem causar em pessoas sem recomendações para uso.

Além disso, Marcon et al.(2012), aponta que o uso indiscriminado, seja para fins terapêuticos ou não acaba gerando problemas como tolerância e dependência. Embora os nootrópicos não se encaixem nessa teoria, seu uso não é inócuo ao ser humano, devendo da mesma forma ser evitado por indivíduos sem prescrição de uso.

\section{CONCLUSÃO}

Este estudo teve o objetivo de avaliar os hábitos de consumo de estimulantes cerebrais entre os futuros profissionais da saúde, em relação à utilização e conhecimento 
dos medicamentos, sobretudo a prática da automedicação. Os profissionais da saúde serão os principais responsáveis em fornecer orientação para a população sobre o uso correto de medicamentos. Sendo o consumo de estimulantes entre os acadêmicos um fato comprovado, a hipótese do projeto é respondida.

Além disso, as universidades devem estar atentas a esses comportamentos de risco, buscando direcionar esses estudantes a aconselhamentos, formando grupos de discussão nas salas de aula, trazendo o tema para o foco atual. O ensino superior exige um grau elevado de comprometimento, ainda mais sobre o fato de que muitos universitários não dispõem de tempo para o estudo, submetendo-se ao uso de estimulantes cerebrais.

Os achados, embora não representem a maioria dos estudantes universitários é um grande ponto de partida para futuros estudos, podendo abranger universidades públicas ou outros cursos de graduação e cursos técnicos. Além disso, sendo o primeiro estudo de forma a abranger diversos medicamentos no Brasil, cria-se a possibilidade de ampliar os questionamentos, analisando a forma com que esses estudantes adquirem essas substâncias ou mesmo a pretensão futura de uso.

\section{REFERÊNCIAS}

ADVOKAT, C.D.; GUIDRY, D.; MARTINO, L. Licit and illicit use of medications for AttentionDeficit Hyperactivity Disorder in undergraduate college students. Journal of American College Health. EUA, v.56, n.6, p.601-606, jun.2008.

ALKURAISHY, M. H.; MAHDI, A. H.; ASHOOR, A. W. Piracetam (2-Oxo-Pyrrolidone) Advance And Modulate Central Processing Reaction Time In Normal Healthy Volunteers: Randomized Experimental Study. Clinical Trail. Iraque, v.3, n.4, p.1-15, abr.2012.

ALKURAISHY, M.H.; ALGAREEB, I.A.; ALBUHADILLY, K.A.; ALMGOTER, M.B. Modulation Effects of Piracetam and Ginkgo Biloba on the Cognitive and Working Memory Functions: Psychometric Study. Neurology and Neurophysiology. Iraque, v.5, n.5, p. 1-6, abr. 2014.

AlóE, F.; ALVES R.C.; ARAÚJO, J.F.; AZEVEDO, A.; BACELAR, A.; BEZERRA, M.; BitTencourt, L. R. A.; BustamANTE, G.; CARDOSO, T. A. M. O.; ECKELI, A. L.; FERNANDES, R. M. F.; GOULART, L., HALLINAN, M.P.; HASAN, R.; SANDER, H.H.; 
PINTO, L.R.J.; LOPES, M. C.; MINHOTO, G. R.; MORAES, W.; MOREIRA, G. A.; PACHITO, D.; PEDRAZOLLI, M.; POYARES, D.; PRADO, L.; RIZZO, G.; RODRIGUES, R. N.; ROITMAN, I.; SILVA, A. B.; TAVARES, S. M. A. Diretrizes brasileiras para o tratamento da narcolepsia. Revista Brasileira de Psiquiatria. São Paulo, v. 32, n. 3, p. 305-314, set. 2010.

AQUINO, S.D.; BARROS, C.A.J.; SILVA, P.D.M. A automedicação e os acadêmicos da área de saúde. Ciência \& Saúde Coletiva. Recife, v.15, n.5, p.2533-2538, 2010.

ARRIA, A.M.; DUPONT, R.L. Nonmedical Prescription Stimulant Use among College Students: Why We Need To Do Something and What We Need To Do. Journal of addictive diseases. Maryland, v.29, n.4, p.417-426, out.2010.

BALBINO, E.E; DIAS, F.M. Farmacovigilância: um passo em direção ao uso racional de plantas medicinais e fitoterápicos. Revista Brasileira de Farmacognosia. Curitiba, v.20, n.6, p.992-1000, out. 2010.

BARROS, D.; ORTEGA, F. Metilfenidato e Aprimoramento Cognitivo Farmacológico: representações sociais de universitários. Saúde e sociedade. Rio de Janeiro, v.20, n.2, p.350-362, 2011.

BATTLEDAY, R.M.; BREM, A.K. Modafinil for cognitive neuroenhancement in healthy nonsleep-deprived subjects: A systematic review. European Neuropsychopharmacology. v.25, n.9, p. 1-17, Ago. 2015.

BRASIL. Agência Nacional de Vigilância Sanitária (ANVISA). Prescrição e consumo de metilfenidato no Brasil: identificando riscos para o monitoramento e controle sanitário. Boletim de Farmacoepidemiologia, v. 2, n.2, p. 1-14, 2012 . Disponível em: http://portal.anvisa.gov.br/wps/wcm/connect/c4038b004e996487ada1 af8a610f4177/ boletim_sngpc_2_2012+corrigido+2.pdf?MOD=AJPERES. Acesso em 08 out. 2015.

CARNEIRO, S.M.; PRADO, A.S.T.; MOURA, H.C.; STRAPASSON, J.F.; RABELO, N.F.; RIBEIRO, T.T.; JESUS, E.C. O uso não prescrito de metilfenidato entre acadêmicos de Medicina. Cadernos UNIFOA. Volta Redonda, v.1, n.1, p.53- 59, mai. 2013. 
CARVALHO, F.R.T.; BRANT, C.L.; MELO, B.M. Exigências de produtividade na escola e no trabalho e o consumo de metilfenidato. Educação e Sociedade. Campinas, v. 35, n. 127, p. 587-604, jun. 2014.

COUTO, S.T.; JUNIOR, M.R.M.; GOMES, A.R.C. Aspectos neurobiológicos do transtorno do déficit de atenção e hiperatividade (TDAH): uma revisão. Ciências e Cognição. Rio de Janeiro, v.15, n.15, p.241-250, abr. 2010.

CUTLER, A.K. Prescription Stimulants Are "A Okay": Applying Neutralization Theory to College Students' Nonmedical Prescription Stimulant Use. Journal of American College Health. Washington, v. 62, n.7, p. 478-486, 2014.

CRUZ, T.C.; JUNIOR, E.P.; GAMA, M.L.; MAIA, L.C.; FILHO, M.J.; NETO, O.M.; COUTINHO, D.M. Uso não-prescrito de metilfenidato entre estudantes de Medicina da Universidade Federal da Bahia. Gazeta Médica da Bahia. Salvador, v. 81, n. 1 p. 3-6, jun.2011.

DECOTELLI, M. K.; BOHRER, T.C.L.; BICALHO, G.P.P. A Droga da Obediência: Medicalização, Infância e Biopoder - Notas Sobre Clínica e Política. Psicologia: Ciência e Profissão, v.33, n.2, p.446-459, 2013.

DESANTIS, A.D.; HANE, A.C. "Adderall is definitely not a drug": justifications for the illegal use of ADHD stimulants. Substance Use and Misuse. Lexington, v.45, n.2, p.31-46, out.2010.

FILHO, C.A; FAKOURY, M.K.; JUNIOR, M.C.W.; FERRY, A.R.F. Ginkgo biloba e Distúrbios de Memória: revisão sistemática. Cadernos Brasileiros de Medicina. v.20, n.1, p.55-62, 2007.

FILHO, C. A.; FAKOURY, M. K.; FERRY, F.R.A. Ginkgo biloba e memória - revisão sistemática. Revista Brasileira de Geriatria e Gerontologia. v. 13, n. 1, p. 145-152, 2010. 
FORLINI, C.; RACINE, E. Disagreements with implications: diverging discourses on the ethics of non-medical use of methylphenidate for performance enhancement. BMC Medicine Ethics, EUA, v.10, n.1, p.1-13, 2009.

FRANCHI, M.J.A.; MACGREGOR, A.; FOND, G. A preliminary study on cognitive enhancer consumption behaviors and motives of French Medicine and Pharmacology students. European Review for Medical and Pharmacological Sciences. Marseille, v.18, n.13, p. 18751878, 2014.

FRANKE, A.G.; BONERTZ, C.; CHRISTMANN, M.; HUSS, M.; FELLGIEBEL, A.; HILDT, E.; LIEB, K. Non-medical use of prescription stimulants and illicit use of stimulants for cognitive enhancement in pupils and students in Germany. Pharmacopsychiatry. Alemanha, v.44, n.2, p.60-66, mar.2011.

FREESE, L.; SIGNOR, L.; MACHADO, C.; FERIGOLO, M.; BARROS, H.M.T. Non-medical use of methylphenidate: a review. Trends Psychiatry Psychother. Porto Alegre, v. 34, n. 2, p. 183-188, 2012.

GIORELLI, S.A.; SANTOS, P.P.; CARNAVAL, T.; GOMES, M.M. Excessive daytime sleepiness: clinical, diagnostic and therapeutic aspects. Revista Brasileira de Neurologia. Rio de Janeiro, v.48, n.3, p.17-24, set. 2012.

GRAÇA, C.S.G. Consumo de estimulantes cerebrais nos estudantes de Medicina da Universidade da Beira Interior. 2013. 50 p. Dissertação (mestrado). Faculdade de Ciências da Saúde, Universidade da Beira Interior, Covilhã, 2013.

GRADY, S.; AESCHBACH, D.; WRIGHT, P.K.; CZEISLER, A.C. Effect of Modafinil on Impairments in Neurobehavioral Performance and Learning Associated with Extended Wakefulness and Circadian Misalignment. Neuropsychopharmacology. Boston, v.35, n. 9, p. 1910-1920, mai. 2010.

HILDT, E.; LIEB, K.; FRANKE, G.A. Life context of pharmacological academic performance enhancement among university students - a qualitative approach. BMC Medical Ethics. Mainz, v.23, n.15, p. 1-10, mar.2014. 
HUSAIN, M.; MEHTA, A.M. Cognitive enhancement by drugs in health and disease Trends in Cognitive Sciences. Londres, v.15, n.1, p. 28-36, jan.2011.

HYMAN, E.S. Cognitive Enhancement: Promises and Perils. NeuroView. Cambridge, v.69, n.4, p.595-598, fev. 2011.

IHL, R. Gingko biloba extract EGb 761: clinical data in dementia .International Psychogeriatrics. Krefeld, v.24, n.1, p. 35-40, ago.2012.

IHL, R. Effects of Ginkgo biloba extract EGb 761 ® in dementia with neuropsychiatric features: review of recently completed randomized, controlled trials. International Journal Psychiatry Clinical Practice. Krefeld, v.17, n.1, p.8-14, ago.2013.

KELLEY, A.M.; WEBB, C.M.; ATHY, J.R.; LEY, S.; GAYDOS, S. Cognition enhancement by modafinil: a meta-analysis. Aviation, Space, and Environmental Medicine. USA, v.83, n.7, p.685-690, jul.2012.

KURZ, C.; UNGERER, I.; LIPKA,U.; KIRR,S.;SCHÜTT,T.; ECKERT,A.; LEUNER,K.; MÜLLER, W.E. The metabolic enhancer piracetam ameliorates the impairment of mitochondrial function and neurite outgrowth induced by B-amyloid peptide. British Journal of Pharmacology. Welwyn Garde, v.160, n.2, p. 246-257, mar. 2010.

LAWS, K. R.; SWEETNAM, H.; KONDEL, T. K. Is Ginkgo biloba a cognitive enhancer in healthy individuals? A meta-analysis. Human Psychopharmacology: Clinical and Experimental. Reino Unido, v. 27, n.6, p. 527-533, nov. 2012.

LEITE, T.C.C.; BRANCO, A. Análise das bulas de medicamentos à base de Ginkgo biloba L. Revista de Ciências Farmacêuticas Básica e Aplicada. Feira de Santana, v.31, n.1, p.8387, mar.2010.

LEUNER, K.; KURZ, C.; GUIDETTI, G.; ORGOGOZO, J.M.; MÜLLER,W.E. Improved Mitochondrial Function in Brain Aging and Alzheimer Disease - the New Mechanism of Action of the Old Metabolic Enhancer Piracetam. Frontiers in Neuroscience. Frankfurt, v. 4, n. 44, p.1-11, mai. 2010. 
MAIER, L.J.; LIECHTI, M.E.; HERZIG, F.; SCHAUB, M.P. To Dope or Not to Dope: Neuroenhancement with Prescription Drugs and Drugs of Abuse among Swiss University Students. Plos one. Zurich, v. 8, n. 11, p. 1-10, nov. 2013.

MALYKH, A.G.; SADAIE, M.R. Piracetam and piracetam-like drugs: from basic science to novel clinical applications to CNS disorders. Drugs. Maryland, v.70, n.3, p.287-312, fev.2010.

MARCON, C.; SILVA, M.A.L.; MORAES, B.M.C; MARTINS, S.J.; CARPES, D.A. Amphetamines use and related substances in contemporary society. Disciplinarum Scientia. Santa Maria, v. 13, n. 2, p. 247-263, jul. 2012.

MOHAMED, A.D.; SAHAKIAN, J.B. The ethics of elective psychopharmacology. The International Journal of Neuropsychopharmacology. Oxford, v.15, n.4, p.559- 571, mai.2012.

MULLER, U.; ROWE, B.J.; RITTMAN, T.; LEWIS, C.; ROBBINS,W.T.; SAHAKIANA, J.B. Effects of modafinil on non-verbal cognition, task enjoyment and creative thinking in healthy volunteers. Neuropharmacology. Cambridge, v.64, n.5, p.490- 494, jul.2013.

ORTEGA, F.; BARROS, D.;CALIMAN, L.; ITABORAHY,C.; JUNQUEIRA,L.; FERREIRA, C. P. Ritalin in Brazil: production, discourse and practices. Interface - Comunicação, saúde, Educação. Rio de Janeiro, v.14, n.34, p.499-510, set.2010.

PARTRIDGE, B.; BELL, S.; LUCKE, J.; HALL, W. Australian university students' attitudes towards the use of prescription stimulants as cognitive enhancers: Perceived patterns of use, efficacy and safety. Drug Alcohol Review. Australia, v.32, n.3, p. 295-302, set.2013.

PASQUINI, N.C. Uso de metilfenidato (MFD) por estudantes universitários com intuito de "turbinar" o cérebro. Revista Brasileira de Biologia e Farmácia. Campina Grande, v. 9, n. 2, p. 107-113, ago.2013.

PEREIRA, A.C.A.I.; BEL, D. E. Metilfenidato: principal tratamento para o Déficit de Atenção e Hiperatividade: características neuroquímicas e seus efeitos em modelos experimentais. 
Neurobiologia. São Paulo, v. 73, n. 2, p. 127-139, ago.2010.

PESSANHA, F.F.; MOTA, S.J. Prevalência do uso de metilfenidato por universitários de Campos dos Goytacazes, RJ. Vértices. Campos dos Goytacazes, v.16, n. 1, p. 77-86, set. 2014.

REPANTIS, D.; SCHLATTMANN, P.; LAISNEY, O.; HEUSER, I. Modafinil and methylphenidate for neuroenhancement in healthy individuals: A systematic review. Pharmacological Research. Berlin, v.62, n.3, p.187-206, set.2010.

SAHAKIAN, J.B.; BRUHL, B.A.; COOK, J.; KILLIKELLY, C.; SAVULICH, G.; PIERCY, T.; HAFIZI, S.; PEREZ, J.; EGEA, F.E.; SUCKLING, J.; JONES, B.P. The impact of neuroscience on society: cognitive enhancement in neuropsychiatric disorders and in healthy people. Philosophical Transactions b. Cambridge, v.370, n.1677, p.1-13, ago.2015.

SANDERSLEBEN, V.U.H.; ROTHENBERGER , A.; ALBRECHT,B.; ROTHENBERGER L.G.; KLEMENT, S.; BOCK, N. Ginkgo biloba Extract EGb 761 in Children with ADHD: Preliminary Findings of an Open Multilevel Dose-Finding Study. Zeitschrift für Kinder und Jugendpsychiatrie und Psychotherapie. Berlin, v.42, n. 5, p. 337-347, ago. 2014.

SANIOTIS, A.; HENNEBERG, M.; KUMARATILAKE, J.; GRANTHAM, J. P. Messing with the mind": evolutionary challenges to human brain augmentation. Frontiers in Systems Neuroscience. EUA, v.8, n.152, p.1-6, set. 2014.

SINGH, I., BARD, I. The Smart Drugs Study. London School of Economics and Political Science 2012. Disponível em: http://www.thesmartdrugstudy.com/. Acesso em: 15 out.2015.

SINGH, I.; BARD, I., JACKSON, J. Robust Resilience and Substantial Interest: A Survey of Pharmacological Cognitive Enhancement among University Students in the UK and Ireland. Plos One. Reino Unido, v.9, n.10, p. 1-12, 2014.

SILVA, P. Farmacologia. 7. ed . Rio de Janeiro: Guanabara Koogan, 2010. 1325 p. SMITH, M. E.; FARAH, M. J. Are Prescription Stimulants "Smart Pills"?: The Epidemiology and 
Cognitive Neuroscience of Prescription Stimulant Use by Normal Healthy Individuals. Psychological Bulletin. Pensilvânia, v.137, n. 5, p. 717-741, set. 2011.

SWANSON, J.M.; WIGAL, T.L.; VOLKOW, N.D. Contrast of Medical and Nonmedical Use of Stimulant Drugs, Basis for the Distinction, and Risk of Addiction: Comment on Smith and Farah (2011). Psychological bulletin. Florida, v.137, n.5, p.742-748, set.2011.

TEIXEIRA, M. Notícia preliminar sobre uma tendência contemporânea: o "Aperfeiçoamento cognitivo", do ponto de vista da pesquisa em neurociências. Revista Latinoamericana de Psicopatologia Fundamental. São Paulo, v. 10, n. 3, p. 495-503, set. 2007.

TETER, C.J.; MCCABE, S.E.; LAGRANGE, K.; CRANFORD, J.A.; BOYD, C.J. Illicit Use of Specific Prescription Stimulants Among College Students: Prevalence, Motives, and Routes of Administration. Pharmacotherapy. Florida, v.26, n.10, p.1501-1510, out. 2010.

URBAN, K.R.; GAO, W.J. Performance enhancement at the cost of potential brain plasticity: neural ramifications of nootropic drugs in the healthy developing brain. Frontiers Systems Neuroscience. USA, v.8, n. 38,p.30-38,2014.

VRECKO, S. Just How Cognitive Is "Cognitive Enhancement"? On the Significance of Emotions in University Students' Experiences with Study Drugs. AJOB Neuroscience. Londres, v. 4, n.1, p. 4-12, jan. 2013.

VRECKO, S. Everyday drug diversions: A qualitative study of the illicit exchange and nonmedical use of prescription stimulants on a university campus. Social Science and Medicine. Londres, v. 131, n.1, p. 297-304, abr. 2015.

WILENS, T. E.; ADLER, L. A.; ADAMS, J. ; SGAMBATI, E.; ROTROSEN, J.; SAWTELLE, R.; UTZINGER, L.; FUSILLO, S. Misuse and diversion of stimulants prescribed for ADHD: a systematic review of the literature. Journal of the American Academy of Child and Adolescent Psychiatry. Boston, v. 47, n.1, p. 21-31, Jan. 2008. 